\title{
Rapid Screening of Butyl Paraben Additive in Toner Sample by Molecularly Imprinted Photonic Crystal
}

\author{
Yangyang Liu ${ }^{1}$, Hang Gu ${ }^{1,2}$, Jiahua He ${ }^{1}$, Anqi Cui ${ }^{1}$, Xiaoyi $\mathrm{Wu}^{1}{ }^{1}$, Jiaping Lai ${ }^{3, *}$ and Hui Sun ${ }^{1, *}$ \\ 1 School of Environmental Science and Engineering, Guangzhou University, Guangzhou 510006, China; \\ 3220215206@bit.edu.cn (Y.L.); guhang@mail2.sysu.edu.cn (H.G.); hejh@iqtenet.cn (J.H.); \\ 2112004031@e.gzhu.edu.cn (A.C.); 2112004007@e.gzhu.edu.cn (X.W.) \\ 2 School of Environmental Science and Engineering, Sun Yat-sen University, Guangzhou 510006, China \\ 3 School of Chemistry, South China Normal University, Guangzhou 510006, China \\ * Correspondence: laijp@scnu.edu.cn (J.L.); esesunhui@gzhu.edu.cn (H.S.)
}

check for updates

Citation: Liu, Y.; Gu, H.; He, J.; Cui, A.; Wu, X.; Lai, J.; Sun, H. Rapid Screening of Butyl Paraben Additive in Toner Sample by Molecularly Imprinted Photonic Crystal. Chemosensors 2021, 9, 314. https://doi.org/10.3390/ chemosensors 9110314

Academic Editor:

Nicole Jaffrezic-Renault

Received: 2 September 2021

Accepted: 24 October 2021

Published: 6 November 2021

Publisher's Note: MDPI stays neutral with regard to jurisdictional claims in published maps and institutional affiliations.

Copyright: (c) 2021 by the authors. Licensee MDPI, Basel, Switzerland. This article is an open access article distributed under the terms and conditions of the Creative Commons Attribution (CC BY) license (https:/ / creativecommons.org/licenses/by/ $4.0 /)$.

\begin{abstract}
In consideration of the endocrine disrupting effects caused by the butyl paraben (BP), a portable visual sensor has been developed based on the photonic crystal and molecular imprinting technology for the rapid screen of BP in toner sample which is a type of aqueous cosmetic to soften the face skin. By integrating the self-reporting and molecular recognition properties, the molecular imprinting photonic crystal (MIPC) sensor can display obvious color changes regularly according to the concentration of BP. Based on the "color guide", the content of BP in toner sample can be estimated directly with the naked eye. In addition, the Bragg diffraction spectrum of MIPC can red shift linearly with the increase of the concentration of BP in sample solution with correlation coefficient as 0.9968 . The quantitative determination of BP can be achieved through the optical fiber spectrometer with detection limit as $0.022 \mathrm{mmol} \cdot \mathrm{L}^{-1}$. With good selectivity, this MIPC film can recognize BPs against the complex sample matrix, showing a standard addition recovery of $107 \%$ for the real samples.
\end{abstract}

Keywords: photonic crystal; molecular imprinting technology; estrogen; preservative; butyl p-hydroxybenzoate

\section{Introduction}

Butyl paraben (BP) has been widely used in humanity's daily life. As the antimicrobial additive, it can be found in cosmetics, pharmaceuticals, as well as food products [1-3]. In consideration of the estrogenic effects caused by parabens, the application of parabens has aroused the increasing concerns on the latent hazards to health and environment $[4,5]$. It has been demonstrated that the chronic exposure to BP can cause breast cancer and breast tumors, as well as the impairment of the sperm quality and fecundity [6-10]. The European Union and China have stipulated the limit for BP at concentrations up to $0.14 \%$ $(\mathrm{g} / \mathrm{g})$ as the preservative in cosmetic products, including lotion, toilet water, face creams and rinse-off products, and so on [11].

Currently, some feasible methods have been applied for the BP detection, e.g., gas chromatography-mass spectrometry method (GC-MS) [12], high performance liquid chromatography (HPLC) [13], capillary electrophoresis (CE) [14], liquid chromatography -mass spectrometry (LC-MS) [15], and high-performance-liquid chromatography-photodiode array analysis (HPLC-PDA) [16,17] etc. Although these methods are sensitive and have wide measurement ranges, most of them need the expensive instruments, complicated sample preconcentration steps, as well as the experienced technicians, which are challenges for the real-time and rapid detection on the spot. Since the international trade is playing an increasingly important role, it is necessary to develop a sensor for the rapid spot screen of BP in the complicated samples. Especially, the portable visual sensor will be more welcome, because it can provide the intuitive and reasonable signal on-site. 
The molecular imprinting polymers (MIPs) was proposed by Wulff in 1972. In the technique, the monomer(s) are frozen in polymeric structures with the presence of the template molecule [18-20]. After the removal of template molecule, the cavities complementary to the template in shape and binding sites are formed. Except specific recognition, the superiorities of the MIPs, such as easy preparation, physical robustness, low-cost, and thermal stability have resulted in increased researcher attraction [19-21]. MIPs have been widely employed in various areas, such as chromatographic separation, catalysis, and sensor platforms. Owing to the intuitiveness, different kind of colorimetric detection platforms have been developed by coupling with MIPs. For example, the MIPbased fluorescent sensor, enzyme assisted colorimetric method, and surface-enhanced Raman scattering (SERS), have been widely applied in clinic detection, food analysis, and environmental monitoring [22-25].

The photonic crystal (PC) materials have drawn increasing interests for endowing the MIPs with the self-reporting properties. PC is composed of several kinds of the regular shaped materials which have different dielectric constants and are periodically arranged in the PC skeleton [26-28]. Owing to the existence of photonic band gap in the PCs, only the light with certain wavelengths can be reflected from their surfaces, resulting in the characteristic structural colors of the PCs [29-31]. Any slight change of the lattice constant of the PC skeleton caused by the stimuli, such as the acidity, temperature, humidity, ionic strength as well as the concentration/speciation variations of the chemicals, will result in the shift of the Bragg reflection peak. And the shift of the Bragg reflection peak will consequently make the PC display the different colors according to the migration degree of the Bragg reflection peak [32]. The composite of PCs and MIPs, known as the MIPC, can integrate the advantages of both [33-35]. With the ability to transfer the molecular recognition process specifically into the spectral signals, MIPCs can be used to sense the targeted molecules visually, without needing the complex label techniques and expensive instruments. The MIPCs have been applied successfully in many fields such as poison analysis, clinical diagnosis, virus detection, customs security, and other fields [36-40].

In the present work, an MIPC sensor has been fabricated for the visual detection of $\mathrm{BP}$ in toner sample without sample pre-treatment. It can sense the trace BP with high sensitivity. Owing to the obvious regular color changes of MIPCs during the molecular recognition process, a "color guide" has been fabricated. The content of BPs in toner samples can be estimated by comparing the actual colors of the MIPCs with the "color guide". Furthermore, the accurate concentration of BP can be obtained through the optical fiber spectrometer, since the shift value $(\Delta \lambda)$ of the Bragg diffraction peak is positively correlated with the BP concentration. The factors that affect the analytical performance have been optimized and assessed.

\section{Materials and Methods}

\subsection{Materials}

Butyl paraben (BP, CAS 94-26-8), methyl paraben (MP, CAS 99-76-3), tetraethyl orthosilicate (TEOS, CAS 78-10-4), procaine (CAS 51-05-8), and benzocaine (CAS 9409-7) were purchased from Aladdin (Shanghai, China). Acrylic acid (AA, CAS 79-107), 2, 2-Azobisisobutyronitrile (AIBN, CAS 78-67-1), and methacrylic acid (MAA, CAS 79-41-4) were obtained from Tianjin Damao chemical reagent factory (Tianjin, China). Hydrochloric acid (CAS 7647-01-0), absolute ethyl alcohol (CAS 64-17-5), glacial acetic acid (CAS 64-19-7), concentrated sulfuric acid $\left(\mathrm{H}_{2} \mathrm{SO}_{4}, 98 \%\right.$, CAS 7664-93-9), and 25\% ammonia water $\left(\mathrm{NH}_{3} \cdot \mathrm{H}_{2} \mathrm{O}\right.$, CAS 1336-21-6) were purchased from Tianjin Zhiyuan chemical reagent Co., Ltd (Tianjin, China). Further, 30\% hydrogen peroxide (CAS 7722-84-1) and $45 \%$ hydrofluoric acid (CAS 7664-39-3) were purchased from the Guangzhou chemical reagent factory (Guangzhou, China). Ethylene dimethacrylate (EDMA, CAS 97-90-5) was purchased from Alfa Aesar chemical Co., Ltd (Shanghai, China). Hydroxyisobutyric acid (CAS 594-61-6) was purchased from Shanghai Renxi technology Co., Ltd (Shanghai, China). All of the above chemicals were of analytical grade. Ultrapure water $(18.2 \mathrm{M} \Omega \cdot \mathrm{cm})$ 
was used in all solutions. The transparent soda-lime glass $\left(\mathrm{Na}_{2} \mathrm{O} \cdot \mathrm{CaO} \cdot 6 \mathrm{SiO}_{2}\right)$ slides $\left(50 \times 9 \times 1 \mathrm{~mm}^{3}\right)$ were purchased from Jiangsu swift boat glass and plastic Co., Ltd (Yancheng, China). Additionally, polymethyl methacrylate (PMMA) slides $(50 \times 12 \times$ $1.2 \mathrm{~mm}^{3}$ ) were used directly without any treatment. The PMMA slides were purchased from Yali organic process factory (Guangzhou, China).

\subsection{Apparatus}

The silica colloidal microspheres were collected by a centrifuging apparatus (TG18WS, Hunan Xiangli instrument Co., Ltd. Changsha, China). Maya 2000 PRO fiber optic spectrometer (Ocean Optics, Orlando, FL, USA) with haloid lamp cold-light source (LS3000, 360-2000 nm) was used to record the diffraction spectra. The self-assembly of the $\mathrm{SiO}_{2}$ based PC template was conducted by using a constant temperature and humidity chamber (WS-01Y, Hengfeng medical equipment Co. LTD, Huangshi, China). The JSM7001F field emission scanning electron microscope (Hitachi, Tokyo, Japan) was used for getting the SEM images. The HPLC analysis was performed with Shimadzu LC-20 AD with DAD detector (SPDM20A) (Tokyo, Japan).

\subsection{Experimental Methods}

2.3.1. Preparation and Self-Assembly of Silica Colloidal Microspheres

Anhydrous ethanol $(16.25 \mathrm{~mL})$, deionized water $(24.75 \mathrm{~mL})$, and aqueous ammonia $(25 \%, 6.75 \mathrm{~mL})$ were mixed in a conical flask and stirred at $1100 \mathrm{r} / \mathrm{min}$ for $5 \mathrm{~min}$ at room temperature. Next, $45.5 \mathrm{~mL}$ anhydrous ethanol and $6.75 \mathrm{~mL}$ tetraethyl orthosilicate (TEOS) were added. After stirring at $1100 \mathrm{r} / \mathrm{min}$ for $1 \mathrm{~min}$, the speed was reset at $400 \mathrm{r} / \mathrm{min}$ for $2 \mathrm{~h}$. Then, the resulted $\mathrm{SiO}_{2}$ particles were collected by centrifugation and rinsed five times with anhydrous ethanol. Subsequently, these silica particles were dispersed in anhydrous ethanol, and stored in vials in different weight concentration separately.

Basing on the $\mathrm{SiO}_{2}$, the PC films were fabricated on glass substrates through vertical deposition. The detailed procedures are as follows: firstly, the glass substrates were soaked in $\mathrm{H}_{2} \mathrm{SO}_{4} / \mathrm{H}_{2} \mathrm{O}_{2}(7 / 3, v / v)$ solution for $12 \mathrm{~h}$ to clean the glass plates and make them become hydrophilic. After being rinsed with ultrasonic water bath for $1 \mathrm{~h}$, they were immersed vertically in the glass bottles which contained appropriate amount of $\mathrm{SiO}_{2}$ colloidal solution $\left(8 \mathrm{mg} \cdot \mathrm{mL}^{-1}\right)$. Then, the bottles were putted in an incubator with temperature at $30{ }^{\circ} \mathrm{C}$ and humidity at $40 \%$. With the evaporation of the solution, the $\mathrm{SiO}_{2}$ was self-assembled uniformly on the glass plate, resulting in the PC template with obvious structure color.

\subsubsection{Fabrication of the Molecularly Imprinted Photonic Crystal (MIPC)}

The fabrication approach (Scheme 1) is as following: First, $4 \mathrm{mmol} \mathrm{AA}$ and $1 \mathrm{mmol} \mathrm{BP}$ were dissolved with water/methanol $(3: 5, v / v)$. After storing overnight at $4{ }^{\circ} \mathrm{C}$, EDMA $(1 \mathrm{mmol})$ and AIBN (0.003 $\mathrm{g}$ ) were added in the mixture. The resulted precursor solution was then degassed with nitrogen for several minutes to remove oxygen. In the meantime, a "sandwich" was prepared by sandwiching the PC template between two PMMA slides. Then, $10 \mu \mathrm{L}$ precursor liquid was injected into the "sandwich" gaps gently. After that, the "sandwich" was put in the airtight container $\left(60^{\circ} \mathrm{C}\right)$ for $6 \mathrm{~h}$ for MIP polymerization. 


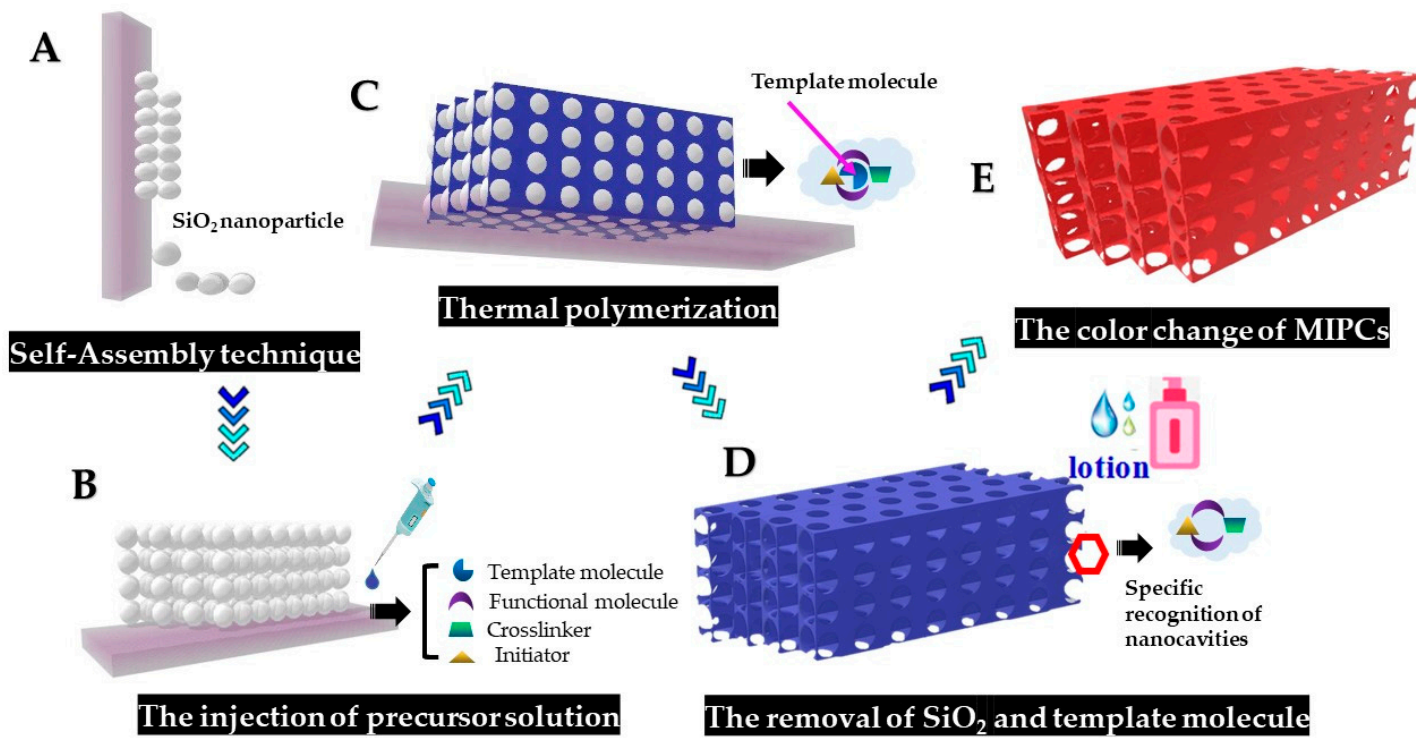

Scheme 1. The schematic for the fabrication and application of the MIPC. (A) fabrication of PC template by self-assembly of silicon spheres; (B) injection of MIP precursor solution on the PC template; (C) thermal polymerization of BP imprinted membrane; (D) preparation of inverse opal MIPC via eliminating of the silica spheres and BP molecules; and (E) the utilization of MIPC in BP monitoring.

Next, the "sandwich" was immersed in 1\% hydrofluoric acid until the glass and PMMA slides were separated from each other. The obtained PMMA slides were then immersed in $4 \%$ hydrofluoric acid for 5 min to remove the $\mathrm{SiO}_{2}$ completely. This way, the molecular imprinted film with the inverse opal structure was formed on the PMMA slides. Then, the PMMA slides were subjected to the elution repeatedly with a mixture of methanol/water/acetic acid/hydrochloric acid (3.5:5.6:0.5:0.4, $v / v / v / v)$ until no BP was detected in the eluent at $258 \mathrm{~nm}$ by using an UV spectrometer (Jinghua Technology Instrument Co. LTD, Shanghai, China).

\subsection{Investigation of the Analytical Performance of the MIPCs}

To investigate the effect of sample matrix on the sensing performance, different amount of methanol was mixed in the sample matrix with the final content changing from $10 \%$ to $30 \%(v / v)$. The corresponding diffraction spectra were recorded with fiber optic spectrometer.

To explore the response time and the reusability of MIPCs, the same piece of MIPC film was subjected to the adsorption and elution repeatedly, and the corresponding diffraction spectra were recorded.

Benzocaine, procaine and methyl paraben solution with concentrations ranging from 0.0001 to $0.003 \mathrm{~mol} \cdot \mathrm{L}^{-1}$ were prepared. The responses of the MIPC films to these structural analogues of $\mathrm{BP}$ were tested and compared to investigate the molecular recognition properties of the MIPCs.

\subsection{The Application of MIPCs}

A band of alcohol-free rose-water toner, which belongs to the skincare product, was bought from the supermarket. For the toner used in this work, the main ingredients are water, rose oil, nicotinamide and seaweed extract. It can be sprayed on the face for making the skin soft. Since it is non-viscous and almost transparent liquid, so no sample pretreatment procedures have been conducted. Before detection, some methanol was added in the toner to make methanol content achieve $20 \%$. The MIPC film was dipped into the sample solution for $15 \mathrm{~min}$, then the Bragg diffraction of the MIPC film was recorded by the optical fiber spectrometer, and the color change of the MIPC was recorded by a 
camera. The sensing performance of MIPC film was evaluated based on the peak shifts of the Bragg diffraction in response to the $\mathrm{BP}$ in real sample solution.

\section{Results}

Scheme 1 describes the overall procedures concerning the fabrication and application of BP MIPCs: (A) fabrication of PC template by self-assembly of silicon spheres; (B) injection of MIP precursor solution on the PC template; (C) thermal polymerization of BP imprinted membrane; (D) preparation of inverse opal MIPC via eliminating of the silica spheres and BP molecules; and (E) the utilization of MIPC in BP monitoring. Each step has been optimized in order to get the MIPCs with excellent properties, such as the acute color indication, fast response, high selectivity, and good reusability.

\subsection{The Fabrication of PC Template and MIPC Sensor}

Before the fabrication of a PC template, it is important to treat the glass plate with $\mathrm{H}_{2} \mathrm{SO}_{4} / \mathrm{H}_{2} \mathrm{O}_{2}$ solution to make the glass plate hydrophilic and clean. On this kind of glass plate, the $\mathrm{SiO}_{2}$ colloidal solution can be driven evenly to climb along the glass plate by the capillary action. Then, with the evaporation of the solvent, the $\mathrm{SiO}_{2}$ can be orderly arranged on the glass plate, and form the PC template with good quality.

Since the optical performance of $\mathrm{PC}$ is determined by the mono-dispersity and particle size of $\mathrm{SiO}_{2}$, the procedures for the fabrication of $\mathrm{SiO}_{2}$ should be optimized first. The Stöber method was modified to prepare the $\mathrm{SiO}_{2}$ microspheres. The purity of TEOS, the proportion of ammonia, ethanol, and TEOS, and even the formula for mixing TEOS with ammonia have been found to affect the shape, particle size, and mono-dispersity of silicon spheres in the previous work $[40,41]$. The proportion of TEOS showed positive effect on the particle size of the silicon sphere. It can be explained as the following: the more TEOS is used, the more silicic acid will be released through the hydrolyzation of TEOS. And the particle size of silica will increase accordingly owing to the subsequent condensation reaction of silicic acid. By changing the synthesis conditions, $\mathrm{SiO}_{2}$ particles with different size were produced. Further, the corresponding PCs were fabricated based on these $\mathrm{SiO}_{2}$ particles. The wavelength of the Bragg diffraction peaks $\left(\lambda_{\max }\right)$ of PC is found positively correlated with the $\mathrm{SiO}_{2}$ particle size (d) and shows a linear correlation $\left(\lambda_{\max }=2.32 \mathrm{~d}\right)$ with $\mathrm{R}^{2}$ as 0.95481 in the present work (Figure S1).

To achieve the naked-eye detection, the wavelength of the diffraction peaks of MIPCs needs to vary evidently in the different visible light regions $(380-780 \mathrm{~nm}$ ) during the sample responding. The MIPCs with structural color between green and yellow are preferable in the present work. As this kind of MIPCs is expected to produce the obvious visual color changes, no matter the color will red shift or blue shift in response to the target analytes.

In order to obtain the ideal MIPCs, the mono-dispersed $\mathrm{SiO}_{2}$ with average particle size of $365 \mathrm{~nm}$ and polydispersity index of 0.019 was selected to fabricate the PCs. The resulted PCs can display red color with the $\lambda_{\max }$ of the Bragg diffraction at $750 \mathrm{~nm}$ (Figure 1a). As shown in the SEM picture (Figure 1a), the monodispersed silica nanoparticles are organized uniformly on the glass plate, producing the face-centered cubic (FCC) lattice. As there are many silicon spheres arranged on the surface, this morphology is vividly called "opal structure". 


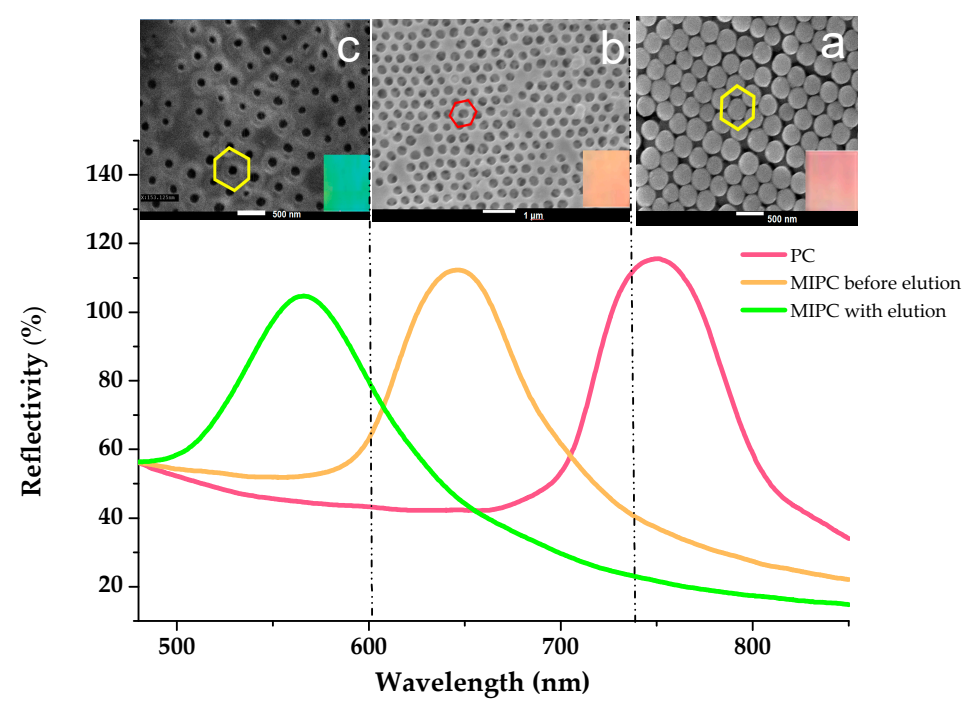

Figure 1. The Bragg diffraction, SEM images and colors (the inset pictures) of PC and MIPC. (a) PC, (b) MIPC before elution of BP, (c) MIPC after elution of BP.

After the polymerization of the precursor on the PC template, MIPs can form in the gap of silicon spheres. Then, through treating with $\mathrm{HF}$, the $\mathrm{SiO}_{2}$ microspheres can be dissolved, resulting in the holes at the original position of these $\mathrm{SiO}_{2}$ microspheres in the MIP film as shown in Figure 1b. This kind of morphology is consequently called "inverse opal structure". By this way, the shape and the arrangement of the $\mathrm{SiO}_{2}$ microspheres can be imprinted into the MIP film. Since the holes can keep the FCC arrangement, the MIP film can display the opal characteristics of the original PC lattice. Consequently, the MIP films are called MIPC.

Since the diameter of the hole in MIPCs is smaller than the original $\mathrm{SiO}_{2}$ sphere owing to the contraction, the corresponding MIPCs will becomes orange with $\lambda_{\max }$ blue-moving to $646 \mathrm{~nm}$. After further elution of the template BP, the molecular recognition sites can be formed on the MIPC film. Owing to the further reduction of diameter of the hole (Figure 1c), the $\lambda_{\max }$ of MIPCs will approach to the boundary between green and yellow, which is $565 \mathrm{~nm}$. It can be seen from Figure 1, even after removing the $\mathrm{SiO}_{2}$ microspheres and the imprinted templates, the inverse opal structure of the resulted MIPC still sustains the FCC arrangement. The periodic variation of refractive index of these porous films can give rise to Bragg optical properties.

The MIPCs accommodate interconnected macropore array, to which nanocavities complementary to BP in shape and binding sites are distributed. The presence of macropores in MIPCs not only provide more interaction sites but also decrease the molecular transport resistance, which is favorable for improving the MIPCs' sensing efficiency. The obtained MIPCs not only have the ideal structural color (between green and yellow), which is conducive to change color in the visible light range, but have the stable and regular microporous structure. These features are helpful for achieving the evident color change in response to the slight shrinkage/swell of the cellular structure of the MIPC during sample monitoring. Thus, in the present study, the $\mathrm{SiO}_{2}$ with particle size of $365 \mathrm{~nm}$ was chosen for the fabrication of PCs and MIPCs.

It is known the recognition sites of MIPCs are resulted from the interactions between the functional monomers and template molecules during the polymerization. The rigidity of the MIPCs, which determines the swell/shrinkage properties of MIPCs, mainly depends on the dosage of the cross-linker used.

To obtain MIPCs with a satisfactory physical and chemical performance, the formula of precursor solution has been optimized in the present work. The insufficient functional monomer will cause the deficient binding with template molecules, but the overdose of functional monomer will produce a large amount of nonspecific binding sites through the 
interaction between the functional monomers during the polymerization. In order to obtain a smart MIPC sensor to convert the molecular recognition process acutely into a visible color change, the network skeleton should have good elasticity and durability, besides the molecular recognition specificity. It should be able to expand and shrink reversibly. Thus, the amount of MAA and EDMA has been investigated. Results (Figure 2a) indicate that the satisfying sensing performance can be obtained when the molar ratio of BP/AA/EDMA is 1:4:1. Thus, this condition has been used for producing the MIPCs in the present work.
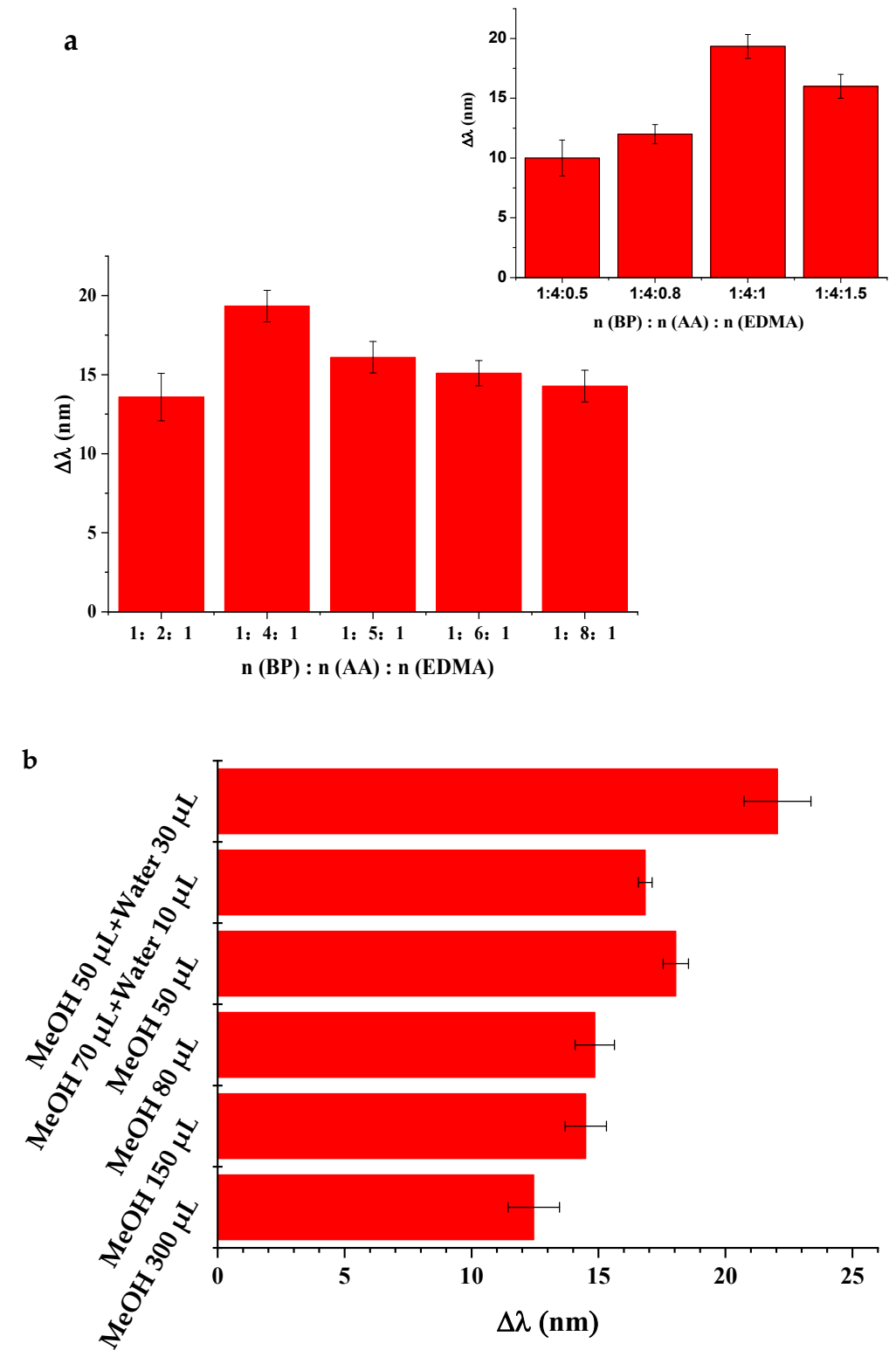

Figure 2. Effect of the molar ratio of BP, AA and EDMA in the precursor solution (a) and the different porogen (b) on the peak shift of MIPCs (The upper and lower figures in Figure 2a indicate respectively the effect of EDMA and MAA on the peak shift of MIPCs; Concentration of BP: $0.001 \mathrm{~mol} \cdot \mathrm{L}^{-1}$ ).

Selecting a proper porogen is also key in order to improve the specific recognition property of MIPCs. It is known that the variation of polarity between the sample solution and the porogen that has been used to synthesize the MIPs will affect the polymer swelling or shrinking, and had a profound effect on the polymer recognition performance [42]. The interaction between the template molecule and the MIPs is governed by different molecular forces in organic solvents and in aqueous sample solution. The interactions that are predominant in the polar environment are mainly the hydrophobic interactions 
and ionic bonds [43]. Since this sensor are expected to be applied in aqueous sample solution, not only should the porogen dissolve BP, functional monomers, and cross-linkers completely, but provide the polarity approximating the real sample solution as closely as possible. Pure methanol was used as the porogen in the preliminary experiment. However, the resulted MIPCs can only display the weak response to BP. In addition, they will crack in the regeneration step, thus shortening the service time. Results (Figure $2 b$ ) indicate that the addition of a proper amount of water in the precursor solution will be helpful to improve the signal $(\Delta \lambda)$ of the corresponding MIPCs in response to the BP sample. Through adjusting the methanol content in the precursor solution, it is found that the response sensitivity of the obtained MIPCs will decrease with the increase of methanol in precursor solution. In the experimental range, when the porogen contains $50 \mu \mathrm{L}$ methanol and $30 \mu \mathrm{L}$ water, the resulted MIPC can display the largest $\Delta \lambda$ value. In order to avoid the further interruption of the hydrogen bond between BP and the functional monomers, the amount of water was not increased continually.

Similar porogen in preparation of MIPs has been used in literature [43]. In that work, the polymerization was conducted in the mixture of methanol/water $(4: 1, v / v)$. For the aqueous sample, the MIPs prepared in the mixed solvent has shown much better recognition properties than in the "classical" way in the presence of nonpolar solvents.

\subsection{Analytical Performance for Sensing BPS}

\subsubsection{Effect of the Methanol Content in the Assayed Sample Solution}

In the pure water samples, the MIPCs can only display the obscure peak-shift, because the hydrogen bond between BPs and the binding sites may be inhibited in the strong polar solution. Since methanol was used in the porogen to prepare the MIPCs in this work, the effect of methanol in the sample matrix on the sensing properties has been studied. Results show (Figure 3a) that the sensing signal can increase gradually with the addition of methanol. The shift $(\Delta \lambda)$ of the diffraction peak can approach the maximum value when $20 \%(v / v)$ methanol is included in the sample matrix. Continue to increase methanol, the $\Delta \lambda$ will decrease instead. Further, in pure methanol, the sensor shows no response at all because of the too high solubility of BPs in methanol. That is, the adsorption performance of MIPCs for BPs is poor when methanol content is high in the sample solution. Attributed to the competitive distribution of BPs between MIPC and solvent phase, the optimal concentration of methanol is $20 \%(v / v)$ in the sample solution. When the same MIPC is inserted in blank samples with methanol content changing from 0 to $30 \%$, no change can be found for the Bragg diffraction.

a

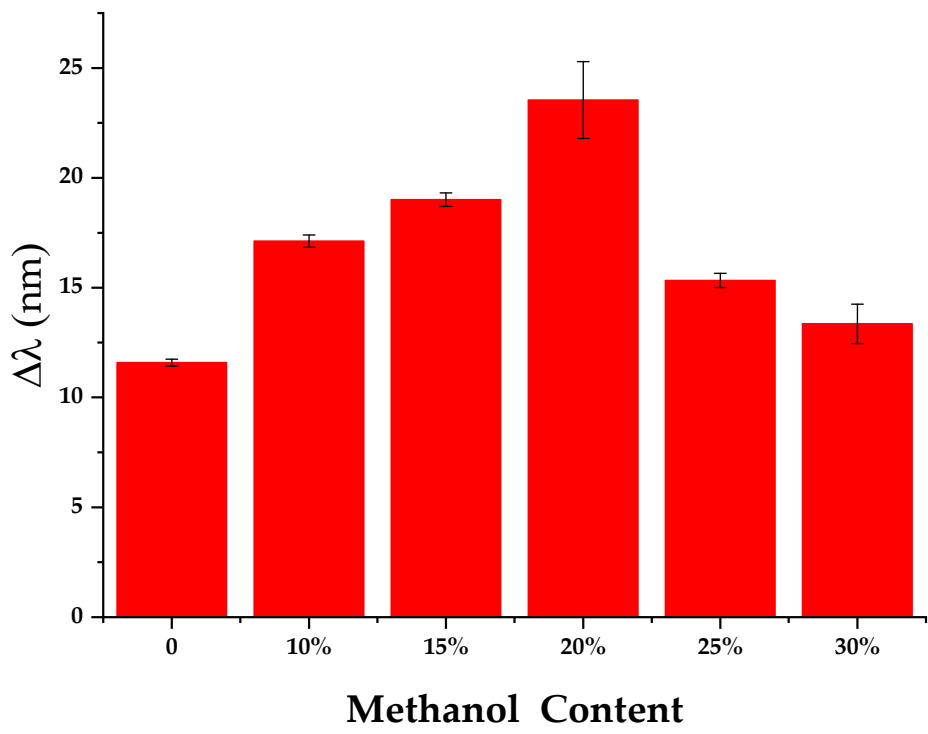

Figure 3. Cont. 

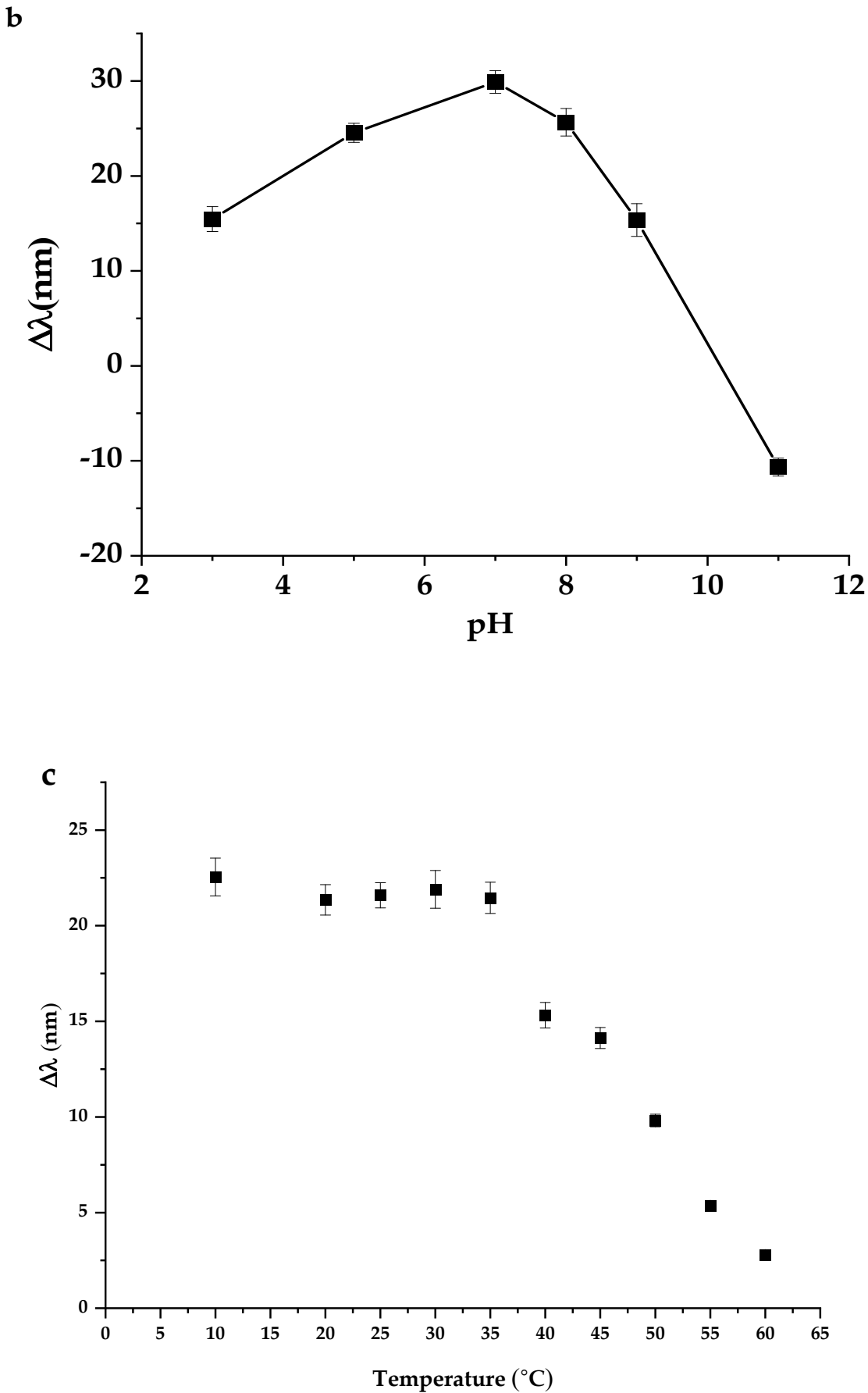

Figure 3. Effect of the methanol content in the assayed sample solution (a), $\mathrm{pH}(\mathbf{b})$ and temperature (c) on the MIPC signal (Concentration of BP: $0.001 \mathrm{~mol} \cdot \mathrm{L}^{-1}$ ).

\subsubsection{The Effect of $\mathrm{pH}$ on the Performance of MIPC}

The effect of $\mathrm{pH}$ on the performance of MIPC has been studied in $0.001 \mathrm{~mol} \cdot \mathrm{L}^{-1} \mathrm{BP}$ solution by using the same piece of MIPC film. The phosphate buffer $(10 \mathrm{mM})$ was used to control the $\mathrm{pH}$ in the present work. From Figure $3 \mathrm{~b}$, it can be seen that the shift value $(\Delta \lambda)$ of the Bragg diffraction peak increases gradually as $\mathrm{pH}$ increases from 3 to 7 , owing to the synergies of the hydrogen bond and the structural complementarity effect between BP and MIPC. However, when the $\mathrm{pH}$ is increased over 8 , the $\Delta \lambda$ of the diffraction peak of the MIPC tends to drop sharply. When $\mathrm{pH}$ value is greater than the $\mathrm{pKa}$ values of $\mathrm{BP}$ (8.41) and acrylic acid (4.26), owing to the acid dissociation effect, the COOH groups on the MIPC skeleton will turn into $-\mathrm{COO}^{-}$completely and the phenolic $-\mathrm{OH}$ of $\mathrm{BP}$ will also become 
the negatively charged form. Thus, the hydrogen bond force between MIPC and BP will be weakened, leading to the decrease of the adsorption capacity for BP. Consequently, the $\Delta \lambda$ of the diffraction peak of the MIPC has been found to decrease with the increase of $\mathrm{pH}$ in alkaline solution. Hence, the MIPCs is suitable to be used in the solution with pH 5-8.

\subsubsection{The Effect of Temperature on the Performance of MIPCs}

To investigate the effect of temperature on the response properties of MIPC membrane, the same piece of MIPC was operated in $0.001 \mathrm{~mol} \cdot \mathrm{L}^{-1} \mathrm{BP}$ solution with the temperature ranging from $10{ }^{\circ} \mathrm{C}$ to $60{ }^{\circ} \mathrm{C}$. It is found that the temperature between $10{ }^{\circ} \mathrm{C}$ and $35{ }^{\circ} \mathrm{C}$ can exert little influence on the performance of MIPCs (Figure 3c). However, when the temperature is over $35^{\circ} \mathrm{C}$, the peak shift $(\Delta \lambda)$ of MIPCs in response to BPs will decrease gradually. When the temperature is higher than $60^{\circ} \mathrm{C}$, the $\Delta \lambda$ approaches to zero, which means the well-aligned inverse opal structure can be demolished by the elevated temperature. Therefore, the MIPC prepared in this experiment is suitable for the practical application between $10^{\circ} \mathrm{C}$ and $35^{\circ} \mathrm{C}$.

\subsubsection{The Analytical Performance of MIPCs to BP}

In BP solution, the analytes can be driven into the MIPC gel to combine with the imprinted binding sites by the concentration difference between the inside and outside the MIPC membrane. Owing to the augment of the ion strength and the swelling of the cavity of the MIPCs, the lattice constant of the PC skeleton will vary considerably along with the shifts of the Bragg diffraction peak. Results reveal that the Bragg diffraction peak of MIPC will red shift gradually with the increases of the soaking time in the sample solution. Then, the $\Delta \lambda_{\max }$ can be achieved when the soaking time approaches $15 \mathrm{~min}$. So, the analytical performance of the MIPCs were evaluated by immersing the same piece of MIPC film into BP solution with different concentration for $15 \mathrm{~min}$. As shown in Figure 4, the wavelength of the Bragg diffraction can increase gradually with the increase of BP concentration. The peak shift $(\Delta \lambda)$ is proportional to the concentration of BPs in the range of $0.1-3.0 \mathrm{mmol} \cdot \mathrm{L}^{-1}$, and the detection limit is $0.022 \mathrm{mmol} \cdot \mathrm{L}^{-1}$ based on three times the ratio of signal/noise.

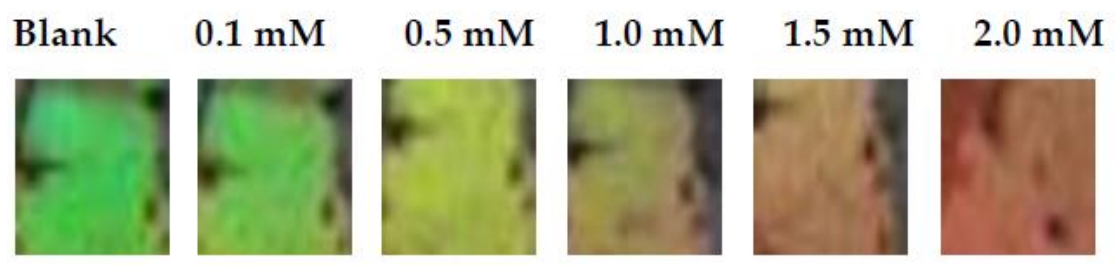

(a)

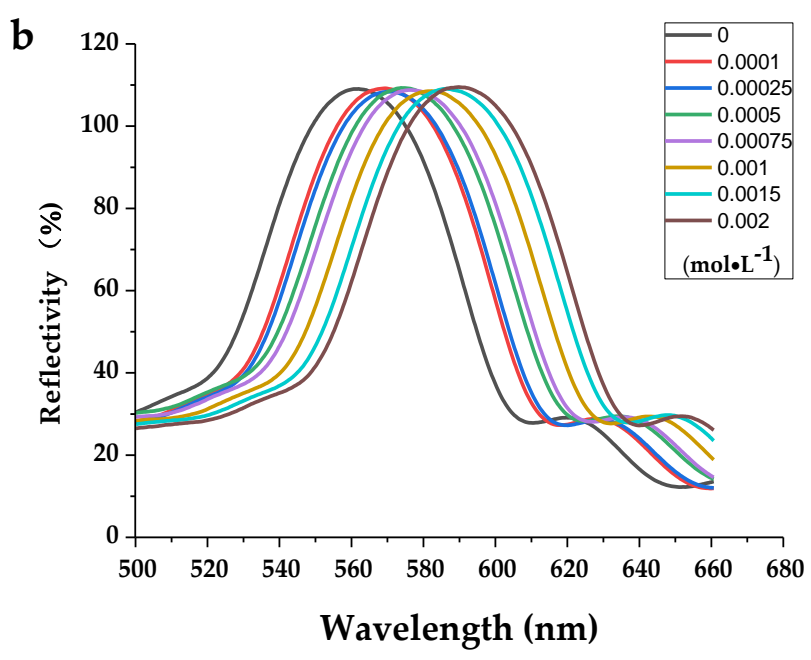

Figure 4. Cont. 


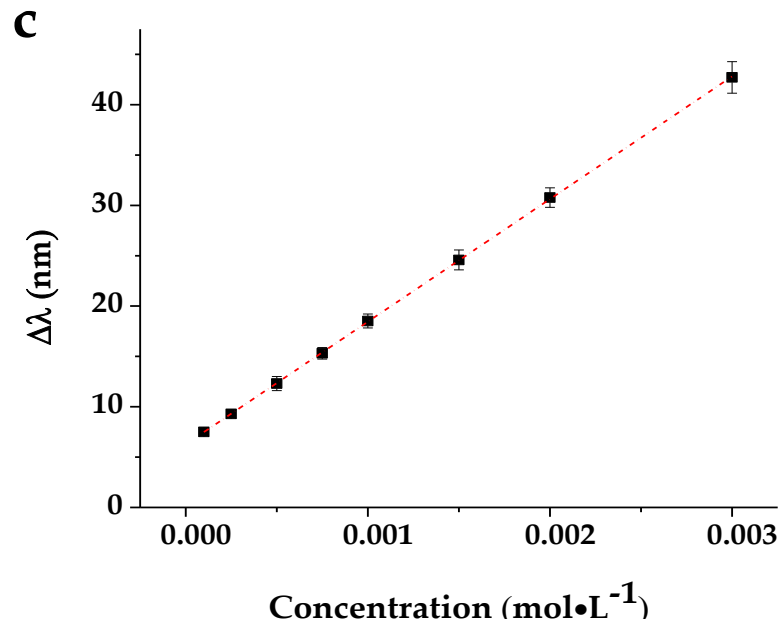

Figure 4. The color changes (a), Bragg diffraction (b), and signal calibration curve (c) of MIPC film upon response to BPs.

In addition to using the calibration curves between $\Delta \lambda$ and the concentration of BP for the quantitation of BP, in the present work, the color changes of the MIPC can also be used to estimate the concentration of the target analytes. In the blank solution the structure color of the MIPC is green. Along with the increase of the BP concentration, the film can display the colors changes regularly from the original green to yellow, and then to red. The pattern of color change can be used as the standard "color guide". Through comparing the actual color of MIPC in the sample solution with the "color guide", we can judge the presence of $\mathrm{BP}$ and estimate the change of BP concentration with the naked eyes, except by using the optical fiber spectrum.

\subsubsection{The Selectivity of MIPCs Film to BP}

As a sensor, the high selectivity is crucial to eliminate the interferences caused by the possible structural analogues. With the similar structure (Figure 5a) to BP, benzocaine, procaine, and methyl paraben (MP) were used to investigate the selectivity of the developed MIPCs. As shown in Figure 5, the response of the MIPC to BP is much stronger than the interferences, which means the MIPCs can recognize BP against these structure analogues.

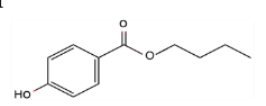

BP

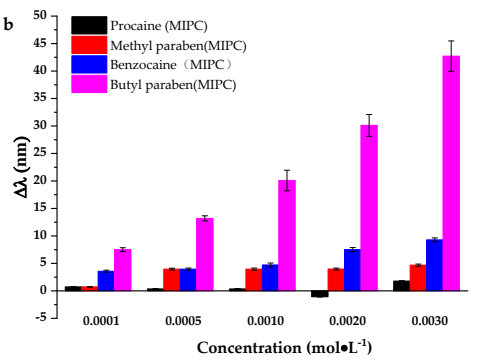

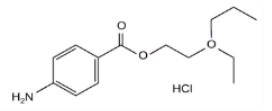

benzocaine

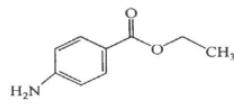

procaine

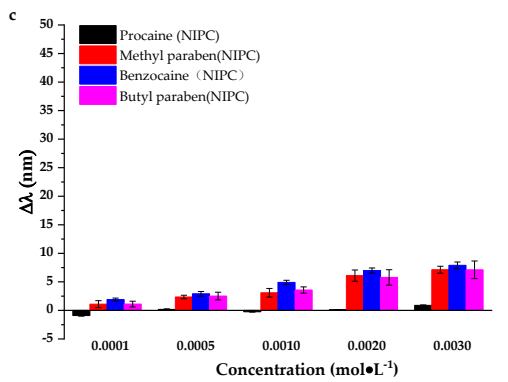

Figure 5. The chemical formula (a) of BP and structural analogues; comparison of the sensing effect of MIPC (b) and NIPC (c) film.

It is known that the signal intensity of MIPC is related to the extent to which the lattice parameters of the PC skeleton can change during the response to analytes. Although methyl 
paraben is expected to nest in the cavity of the MIPCs easier owing to the smaller molecular size, it cannot occupy efficiently the cavity that is designed for the BP with bigger size. The insufficient swelling of the cavity cannot result in the obvious change of the lattice parameters of the PC skeleton. Thus, the response of MIPC to methyl paraben is much poorer than BP (Figure $5 b)$.

Besides methyl paraben, other structure analogues such as benzocaine and procaine have been selected as the interferents in order to further investigate whether the anilne $-\mathrm{NH}_{2}$ group will cause interference. From Figure $5 \mathrm{a}$, it can be seen that benzocaine and procaine have similar structures with paraben. The main difference is that the phenolic $-\mathrm{OH}$ is replaced with anilne $-\mathrm{NH}_{2}$. The selectivity experiment was conducted in the neutral solution ( $\mathrm{pH} 7$ ). In this solution, the $-\mathrm{COOH}$ exist mainly as the negative form, so the acid-base interaction between $-\mathrm{COOH}$ and anilne $-\mathrm{NH}_{2}$ can be ignored. Owing to the imprinting effect and the stronger hydrogen bond interaction between MIPC skeleton and $\mathrm{BP}$, the response of the MIPC to $\mathrm{BP}$ is much stronger than benzocaine and procaine.

Given the outcome procured, it is distinct that the specific recognition sites have been fabricated in the lattice structure of the MIPC films. The MIPC can recognize BP against those with a similar structure.

Under the same conditions, non-imprinted photonic crystals (NIPCs) (Figure 5c) can only show the weak response to all the tested chemicals, and cannot differentiate BP from the interferences. It is demonstrated the imprinting technology has indeed played a positive role in the improvement of the selectivity and adsorption capacity of the PC sensors.

\subsubsection{The Reusability of MIPCs Film}

The reusability is an important indicator for evaluating a sensor. In order to regenerate the MIPCs efficiently without destroying the sensing film, the formula of the eluting solution has been optimized. The mixtures of methanol and acetic acid by 9:1 or 8:2 $(v / v)$ have been commonly used to remove the template molecules in literature $[39,44,45]$. However, these kinds of elution solution showed damaging effects on the MIPCs. After repeated elution with the above solution, the MIPCs will crack, and the intensity of Bragg diffraction peak will decrease obviously and even disappear eventually.

After optimization, the mixture of methanol, water, acetic acid, and $\mathrm{HCl}$ with volume ratio as 3.5:5.5:0.5:0.5 was selected as the eluent in the subsequent experiment. With this eluent, the MIPCs can be regenerated successfully and exhibit a satisfying performance in sample detection. As is shown in Figure 6, after seven cycles of adsorption and elution, the diffraction peak shift of MIPCs can exhibit good repeatability with RSD below 5\%, which indicates that the FCC lattice of MIPCs can be sustained after the repeated elution with the eluent.

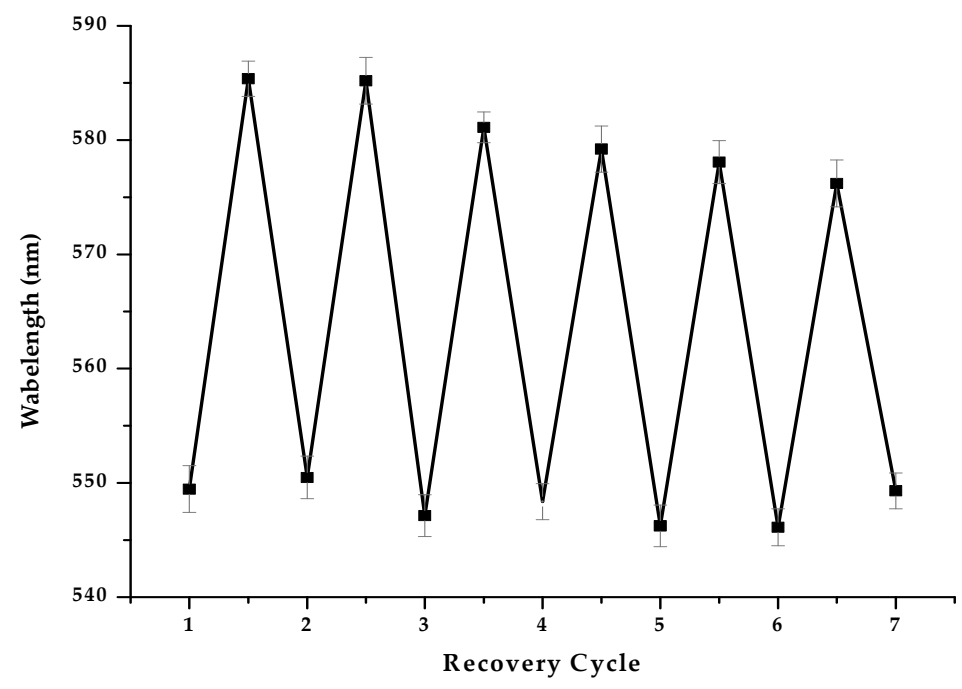

Figure 6. Reusability of the MIPC film. 


\section{Application}

The European Union and China have stipulated the limit for BP in all cosmetic products, such as o/w lotion, toilet water, face creams, and rinse-off products, at concentrations up to $0.14 \%(\mathrm{~g} / \mathrm{g})$ [11]. As to the toner sample used in the present work, the main ingredient is water. Assuming the density of the toner is $1 \mathrm{~g} \cdot \mathrm{mL}^{-1}$, the concentration limit of $0.14 \%$ $(\mathrm{g} / \mathrm{g})$ can be estimated to be $7.2 \times 10^{-3} \mathrm{~mol} \cdot \mathrm{L}^{-1}$ according to the molecular weight of BP. Since the detection limit of the developed method is 325 times lower than the controlled value, the method is sensitive enough to be used for the detection of BPs in real samples. The lotion sample was purchased from the supermarket. No BP was found in the sample by using HPLC. Then the sample was divided into the control group and the spiked group. In the spiked group, $0.0015 \mathrm{~mol} \cdot \mathrm{L}^{-1} \mathrm{BP}$ was added. As shown in Figure 7 , neither the color or the diffraction spectrum of the MIPC changes in the control lotion sample. However, in the spiked lotion sample, a significant peak shift can be found along with the obvious color changing from green to orange for the same MIPC film. Based on the average peak shift value $(\Delta \lambda)$ of $26.1 \mathrm{~nm}(n=5)$, the BP concentration in the spiked sample is calculated to be $0.0016 \mathrm{~mol} \cdot \mathrm{L}^{-1}$ by using the linear regression equation of the MIPC film. In comparison with the spiked value, the standard addition recovery is indicated to be $107 \%$ for the real sample. In addition, though comparing the standard "color guide" with the actual color of MIPC film in the spiked sample solution, the concentration of BP can also be estimated to be about $0.0015 \mathrm{~mol} \cdot \mathrm{L}^{-1}$. Results demonstrate that the developed MIPC can be used as the credible visual sensor to realize the rapid screening and quality estimation for BP in the real samples. Therefore, the visual sensor could become a rapid, reliable, and effective supplement of the optic fiber method in toner samples evaluation.
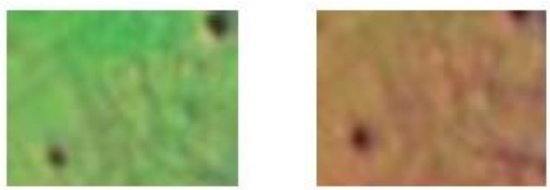

(a)

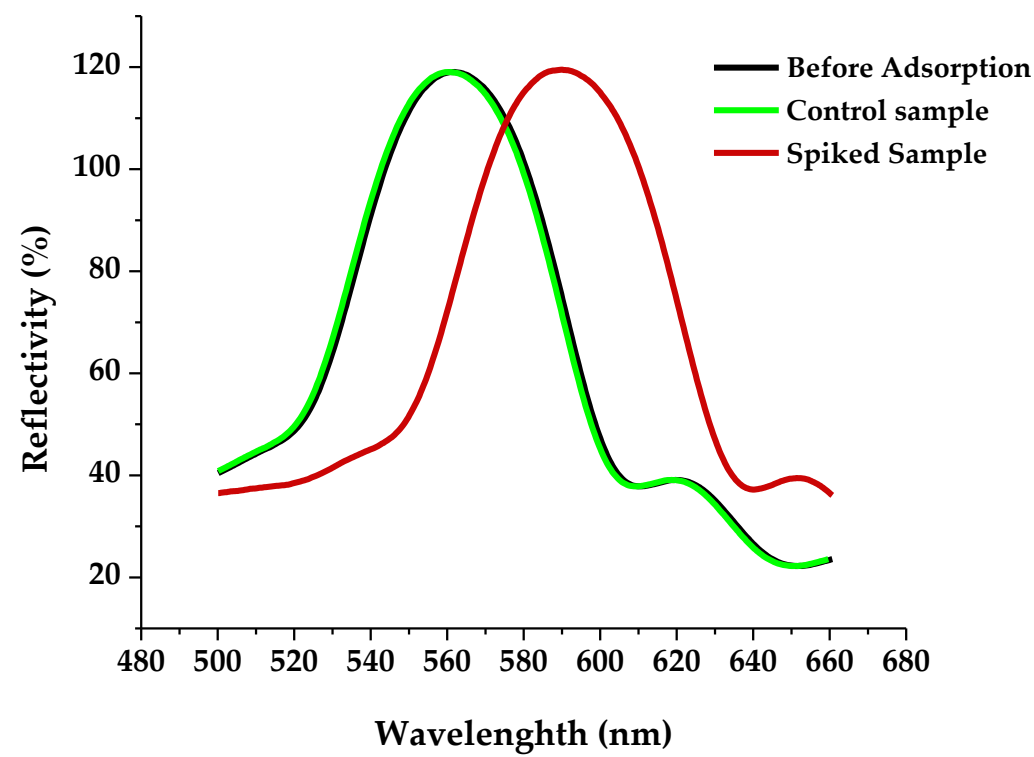

(b)

Figure 7. (a)The color of MIPC in the control sample solution (left, green) and the spiked sample solution (right, orange); (b) The Bragg diffraction spectra of MIPC in different sample solution. 


\section{Conclusions}

A smart visual sensor based on MIPC was developed for the detection of BP in toner samples. In addition to its portability and good reusability, it could provide the intuitive visual signal in response to BP. Through comparing with the "color guide", the content of BPs was estimated rapidly with the naked eye. In addition, the Bragg diffraction spectrum of the MIPC senor red shifted linearly with the increase of the concentration of BPs in sample solution with correlation coefficient as 0.9968 . So, the quantitative detection for BPs was also be achieved. With good selectivity, it recognized BPs against the complex sample matrix, and showed a standard addition recovery of $107 \%$ in the real sample. After further optimization and integrating with the artificial intelligence device, the visual sensor could become a rapid, reliable, and effective supplement of the optic fiber method in the on-site customs inspection.

Supplementary Materials: The following are available online at https:/ /www.mdpi.com/article/ 10.3390/chemosensors9110314/s1, Figure S1: The correlation between the $\mathrm{SiO}_{2}$ diameters and the diffraction peak of MIPC.

Author Contributions: Y.L.: Conceptualization, Writing—original draft, Writing—review and editing. H.G.: Data curation, Methodology, Investigation. J.H.: Methodology, Visualization. A.C.: Validation. X.W.: Resources. J.L.: Supervision, Project administration. H.S.: Supervision, Project administration, Writing - review and editing. All authors have read and agreed to the published version of the manuscript.

Funding: This research was funded by he National Natural Science Foundation of China (21677053, 21876033), Scientific Research Projects in Colleges and Universities of Guangzhou Education Bureau (201831803).

Institutional Review Board Statement: Not available.

Informed Consent Statement: Not available.

Data Availability Statement: Data is contained within the article or supplementary material. All the data will be available to the readers.

Acknowledgments: This work was supported by the National Natural Science Foundation of China (21677053, 21876033), Scientific Research Projects in Colleges and Universities of Guangzhou Education Bureau (201831803).

Conflicts of Interest: The authors declare no conflict of interest.

\section{References}

1. Peinado, F.M.; Lendinez, I.; Sotelo, R.; Iribarne-Duran, L.M.; Fernandez-Parra, J.; Vela-Soria, F.; Olea, N.; Fernandez, M.F.; Freire, C.; Leon, J.; et al. Association of Urinary Levels of Bisphenols A, F, and S with Endometriosis Risk: Preliminary Results of the EndEA Study. Int. J. Environ. Res. Public Health. 2020, 17, 1194. [CrossRef]

2. Gao, C.J.; Kannan, K. Phthalates, bisphenols, parabens, and triclocarban in feminine hygiene products from the United States and their implications for human exposure. Environ. Int. 2020, 136, 105465. [CrossRef] [PubMed]

3. Li, C.; Cui, X.Y.; Chen, Y.; Liao, C.Y. Paraben concentrations in human fingernail and its association with personal care product use. Ecotoxicol. Environ. Saf. 2020, 202, 110933. [CrossRef]

4. Oliveira, M.M.; Martins, F.; Silva, M.G.; Correia, E.; Videira, R.; Peixoto, F. Use of Parabens (Methyl and Butyl) during the Gestation Period: Mitochondrial Bioenergetics of the Testes and Antioxidant Capacity Alterations in Testes and Other Vital Organs of the F1 Generation. Antioxidants 2020, 9, 1302. [CrossRef] [PubMed]

5. Bledzka, D.; Gromadzinska, J.; Wasowicz, W. Parabens. From environmental studies to human health. Environ. Int. 2014, 67, 27-42. [CrossRef] [PubMed]

6. Arya, S.; Dwivedi, A.K.; Alvarado, L.; Kupesic-Plavsic, S. Exposure of U.S. population to endocrine disruptive chemicals (Parabens, Benzophenone-3, Bisphenol-A and Triclosan) and their associations with female infertility. Environ. Pollut. 2020, 265, 114763. [CrossRef] [PubMed]

7. Li, C.; Zhao, Y.; Liu, S.; Yang, D.; Ma, H.; Zhu, Z.; Kang, L.; Lu, S. Exposure of Chinese adult females to parabens from personal care products: Estimation of intake via dermal contact and health risks. Environ. Pollut. 2021, 272, 116043. [CrossRef]

8. Bayulken, D.G.; Tuylu, B.A. In vitro genotoxic and cytotoxic effects of some paraben esters on human peripheral lymphocytes. Drug. Chem. Toxicol. 2019, 42, 386-393. [CrossRef] 
9. Amin, M.M.; Tabatabaeian, M.; Chavoshani, A.; Amjadi, E.; Hashemi, M.; Ebrahimpour, K.; Klishadi, R.; Khazaei, S.; Mansourian, M. Paraben Content in Adjacent Normal-malignant Breast Tissues from Women with Breast Cancer. Biomed. Environ. Sci. 2019, 32, 893-904. [CrossRef]

10. Fransway, A.F.; Fransway, P.J.; Belsito, D.V.; Yiannias, J.A. Paraben Toxicology. Dermatitis 2019, 30, 32-45. [CrossRef]

11. Gardner, S. EU Tightens Restrictions on Three Substances Used in Cosmetics. Infant Care Products. Int. Environ. Rep. 2014, 37, 1362.

12. Kaur, R.; Kaur, R.; Grover, A.; Rani, S.; Malik, A.K.; Kabir, A.; Furton, K.G. Fabric phase sorptive extraction/GC-MS method for rapid determination of broad polarity spectrum multi-class emerging pollutants in various aqueous samples. J. Sep. Sci. 2019, 42, 2407-2417. [CrossRef]

13. Ozcan, S.; Levent, S.; Can, N.O.; Kozanli, M. A Novel HPLC Method for Simultaneous Determination of Methyl, Ethyl, n-propyl, Isopropyl, n-butyl, Isobutyl and Benzyl Paraben in Pharmaceuticals and Cosmetics. Comb. Chem. High Throughput Screen. 2021, 24, 352-365. [CrossRef]

14. Ma, T.; Li, Z.; Jia, Q.; Zhou, W.H. Ultrasound-assisted temperature-controlled ionic liquid emulsification microextraction coupled with capillary electrophoresis for the determination of parabens in personal care products. Electrophoresis 2016, 37, 1624-1631. [CrossRef]

15. Sanchis, Y.; Coscolla, C.; Yusa, V. Analysis of four parabens and bisphenols A, F, S in urine, using dilute and shoot and liquid chromatography coupled to mass spectrometry. Talanta 2019, 202, 42-50. [CrossRef]

16. Alampanos, V.; Kabir, A.; Furton, K.G.; Roje, Z.; Vrcek, I.V.; Samanidou, V. Fabric phase sorptive extraction combined with high-performance-liquid chromatography-photodiode array analysis for the determination of seven parabens in human breast tissues: Application to cancerous and non-cancerous samples. J. Chromatogr. A 2020, 1630, 461530. [CrossRef] [PubMed]

17. Rigkos, G.; Alampanos, V.; Kabir, A.; Furton, K.G.; Roje, Z.; Vrcek, I.V.; Panderi, I.; Samanidou, V. An improved fabric-phase sorptive extraction protocol for the determination of seven parabens in human urine by HPLC-DAD. Biomed. Chromatogr. 2021, 35, e4974. [CrossRef]

18. Chen, L.X.; Wang, X.Y.; Lu, W.H.; Wu, X.Q.; Li, J.H. Molecular imprinting: Perspectives and applications. Chem. Soc. Rev. 2016, 45, 2137-2211. [CrossRef] [PubMed]

19. Alexander, C.; Andersson, H.S.; Andersson, L.I.; Ansell, R.J.; Kirsch, N.; Nicholls, I.A.; O'Mahony, J.; Whitcombe, M.J. Molecular imprinting science and technology: A survey of the literature for the years up to and including 2003. J. Mol. Recognit. 2006, 19, 106-180. [CrossRef]

20. Ashley, J.; Shahbazi, M.A.; Kant, K.; Chidambara, V.A.; Wolff, A.; Bang, D.D.; Sun, Y. Molecularly imprinted polymers for sample preparation and biosensing in food analysis: Progress and perspectives. Biosens. Bioelectron. 2017, 91, 606-615. [CrossRef] [PubMed]

21. Speltini, A.; Scalabrini, A.; Maraschi, F.; Sturini, M.; Profumo, A. Newest applications of molecularly imprinted polymers for exraction of contaminants from environmental and food matrices: A review. Anal. Chim. Acta 2017, 974, 1-26. [CrossRef]

22. Lowdon, J.W.; Eersels, K.; Rogosic, R.; Heidt, B.; Dilien, H.; Redeker, E.S.; Peeters, M.; van Grinsven, B.; Cleij, T.J. Substrate displacement colorimetry for the detection of diarylethylamines. Sens. Actuator B Chem. 2019, 282, 137-144. [CrossRef]

23. Feng, J.W.; Tao, Y.; Shen, X.L.; Jin, H.; Zhou, T.T.; Zhou, Y.S.; Hu, L.Q.; Luo, D.; Mei, S.R.; Lee, Y.I. Highly sensitive and selective fluorescent sensor for tetrabromobisphenol-A in electronic waste samples using molecularly imprinted polymer coated quantum dots. Microchem. J. 2019, 144, 93-101. [CrossRef]

24. Wang, J.X.; Dai, J.D.; Xu, Y.Q.; Dai, X.H.; Zhang, Y.L.; Shi, W.D.; Sellergren, B.; Pan, G.Q. Molecularly Imprinted Fluorescent Test Strip for Direct, Rapid, and Visual Dopamine Detection in Tiny Amount of Biofluid. Small 2019, 15, 9. [CrossRef] [PubMed]

25. Silverio, O.V.; So, R.C.; Elnar, K.J.S.; Malapit, C.A.; Nepomuceno, M.C.M. Development of dieldrin, endosulfan, and hexachlorobenzene-imprinted polymers for dye-displacement array sensing. J. Appl. Polym. Sci. 2017, 134, 11. [CrossRef]

26. Chen, K.; Fu, Q.Q.; Ye, S.Y.; Ge, J.P. Multicolor Printing Using Electric-Field-Responsive and Photocurable Photonic Crystals. Adv. Funct. Mater. 2017, 27, 1702825. [CrossRef]

27. Ge, J.P.; Yin, Y.D. Responsive Photonic Crystals. Angew. Chem. Int. Edit. 2017, 50, 1492-1522. [CrossRef]

28. Hou, J.; Li, M.Z.; Song, Y.L. Recent advances in colloidal photonic crystal sensors: Materials, structures and analysis methods. Nano Today 2018, 22, 132-144. [CrossRef]

29. Wang, H.F.; Gupta, S.K.; Xie, B.Y.; Lu, M.H. Topological photonic crystals: A review. Front Opto-Electron. $2020,13,50-72$. [CrossRef]

30. Joannopoulos, J.D.; Villeneuve, P.R.; Fan, S. Photonic crystals: Putting a new twist on light. Nature 1997, 386, 143-149. [CrossRef]

31. Fenzl, C.; Hirsch, T.; Wolfbeis, O.S. Photonic Crystals for Chemical Sensing and Biosensing. Angew. Chem. Int. Edit. 2014, 53, 3318-3335. [CrossRef]

32. Xu, J.S.; Shang, M.; Liu, J.; Chen, X.; Cao, Y.H. Simultaneous self-assembly of molecularly imprinted magnetic nanoparticles to construct a magnetically responsive photonic crystals sensor for bisphenol A. Sens. Actuator B Chem. 2021, 338, 129858. [CrossRef]

33. Chiappini, A.; Pasquardini, L.; Bossi, A.M. Molecular Imprinted Polymers Coupled to Photonic Structures in Biosensors: The State of Art. Sensors 2020, 20, 5069. [CrossRef]

34. Wang, X.H.; Chen, G.; Dong, Z.Q.; Zhu, Z.G.; Chen, C. Progress in molecular imprinted photonic crystals. J. Mater. Eng. 2020, 48, 60-72. [CrossRef] 
35. Lin, Z.Z.; Li, L.; Fu, G.Y.; Lai, Z.Z.; Peng, A.H.; Huang, Z.Y. Molecularly imprinted polymer-based photonic crystal sensor array for the discrimination of sulfonamides. Anal. Chim. Acta 2020, 1101, 32-40. [CrossRef]

36. Rizvi, A.S.; Murtaza, G.; Yan, D.; Irfan, M.; Xue, M.; Meng, Z.H.; Qu, F. Development of Molecularly Imprinted 2D Photonic Crystal Hydrogel Sensor for Detection of L-Kynurenine in Human Serum. Talanta 2020, 208, 120403. [CrossRef] [PubMed]

37. Qiu, X.Z.; Chen, W.M.; Luo, Y.L.; Wang, Y.L.; Wang, Y.L.; Guo, H.S. Highly sensitive alpha-amanitin sensor based on molecularly imprinted photonic crystals. Anal. Chim. Acta 2020, 1093, 142-149. [CrossRef]

38. Sai, N.; Wu, Y.T.; Sun, Z.; Yu, G.G.; Huang, G.W. A novel photonic sensor for the detection of chloramphenicol. Arab. J. Chem. 2019, 12, 4398-4406. [CrossRef]

39. Hammoud, A.; Chhin, D.; Nguyen, D.K.; Sawan, M. A new molecular imprinted PEDOT glassy carbon electrode for carbamazepine detection. Biosens. Bioelectron. 2021, 180, 113089. [CrossRef]

40. Zhang, Y.; Jin, Z.K.; Zeng, Q.S.; Huang, Y.M.; Gu, H.; He, J.H.; Liu, Y.Y.; Chen, S.L.; Sun, H.; Lai, J.P. Visual test for the presence of the illegal additive ethyl anthranilate by using a photonic crystal test strip. Microchim. Acta 2019, 186, 685. [CrossRef]

41. Chen, S.L.; Sun, H.; Huang, Z.J.; Jin, Z.K.; Fang, S.Y.; He, J.H.; Liu, Y.Y.; Zhang, Y.; Lai, J.P. The visual detection of anesthetics in fish based on an inverse opal photonic crystal sensor Electronic supplementary information (ESI) available. RSC Adv. 2019, 9, 16831-16838. [CrossRef]

42. Turner, N.W.; Piletska, E.V.; Karim, K.; Whitcombe, M.; Malecha, M.; Magan, N.; Baggiani, C.; Piletsky, S.A. Effect of the solvent on recognition properties of molecularly imprinted polymer specific for ochratoxin A. Biosens. Bioelectron. 2004, 20, $1060-1067$. [CrossRef] [PubMed]

43. Haupt, K.; Dzgoev, A.; Mosbach, K. Assay system for the herbicide 2,4-dichlorophenoxyacetic acid using a molecularly imprinted polymer as an artificial recognition element. Anal. Chem. 1998, 70, 628-631. [CrossRef]

44. Fan, J.; Qiu, L.L.; Zheng, W.X.; Meng, Z.H.; Xue, M.; Qiao, Y. Rapid self-assembly preparation of p-nitrophenol-molecular imprinted photonic crystal sensors. Microchem. J. 2021, 164, 105950. [CrossRef]

45. Dehghani, Z.; Akhond, M.; Absalan, G. Carbon quantum dots embedded silica molecular imprinted polymer as a novel and sensitive fluorescent nanoprobe for reproducible enantioselective quantification of naproxen enantiomers. Microchem. J. 2021, 160, 105723. [CrossRef] 